\title{
SYMMETRIES OF KIRCHBERG ALGEBRAS
}

\author{
DAVID J. BENSON, ALEX KUMJIAN, AND N. CHRISTOPHER PHILLIPS
}

\begin{abstract}
Let $G_{0}$ and $G_{1}$ be countable abelian groups. Let $\gamma_{i}$ be an automorphism of $G_{i}$ of order two. Then there exists a unital Kirchberg algebra $A$ satisfying the Universal Coefficient Theorem and with $\left[1_{A}\right]=0$ in $K_{0}(A)$, and an automorphism $\alpha \in \operatorname{Aut}(A)$ of order two, such that $K_{0}(A) \cong G_{0}$, such that $K_{1}(A) \cong G_{1}$, and such that $\alpha_{*}: K_{i}(A) \rightarrow K_{i}(A)$ is $\gamma_{i}$. As a consequence, we prove that every $\mathbb{Z}_{2}$-graded countable module over the representation ring $R\left(\mathbb{Z}_{2}\right)$ of $\mathbb{Z}_{2}$ is isomorphic to the equivariant K-theory $K^{\mathbb{Z}_{2}}(A)$ for some action of $\mathbb{Z}_{2}$ on a unital Kirchberg algebra $A$.

Along the way, we prove that every not necessarily finitely generated $\mathbb{Z}\left[\mathbb{Z}_{2}\right]$ module which is free as a $\mathbb{Z}$-module has a direct sum decomposition with only three kinds of summands, namely $\mathbb{Z}\left[\mathbb{Z}_{2}\right]$ itself and $\mathbb{Z}$ on which the nontrivial element of $\mathbb{Z}_{2}$ acts either trivially or by multiplication by -1 .
\end{abstract}

\section{INTRODUCTION}

Following Definition 4.3.1 of Part 1 of [22], we use the term Kirchberg algebra for a purely infinite simple separable nuclear $\mathrm{C}^{*}$-algebra. In this paper, we prove that any order two automorphism of the K-theory of a unital Kirchberg algebra $A$ satisfying the Universal Coefficient Theorem, and with $\left[1_{A}\right]=0$ in $K_{0}(A)$, lifts to an order two automorphism of $A$.

Recall the classification theorem for unital Kirchberg algebras satisfying the Universal Coefficient Theorem (14]; Theorem 4.2.4 of [19]): if $A$ and $B$ are such algebras, and if $\theta: K_{*}(A) \rightarrow K_{*}(B)$ is a graded isomorphism such that $\theta\left(\left[1_{A}\right]\right)=\left[1_{B}\right]$, then there is an isomorphism $\varphi: A \rightarrow B$ such that $\varphi_{*}=\theta$. The statement also holds with "isomorphism" replaced by "homomorphism" everywhere (14]; in [19] see Theorem 4.1.3 and the proofs of Corollary 4.4.2 and Theorem 4.2.4). Moreover, every pair of countable abelian groups occurs as the K-theory of a unital Kirchberg algebra satisfying the Universal Coefficient Theorem, and the $K_{0}$-class of the identity can be arbitrary (Section 4.4 in Part 1 of [22] or Theorem 5.2 of [9]). Thus, the classification functor is surjective on isomorphism classes in this case.

George Elliott has asked to what extent this functor "splits", in the same sense in which a surjection between abelian groups might (or might not) split. That is: How close can one come to constructing a functor $F$, from the values of the invariant, to Kirchberg algebras satisfying the Universal Coefficient Theorem, such that $K_{*}\left(F\left(G_{*}\right)\right) \cong G_{*}$, etc.? One can't do this exactly. (See Example 1.1 below.) We may then ask for some weaker sort of splitting, or we can try to eliminate the problem by reducing the number of morphisms in the categories. The question

Date: 7 Feb. 2003.

1991 Mathematics Subject Classification. Primary 20C10, 46L55; Secondary 19K99, 19L47, 46L40, 46L80.

Research partially supported by NSF grants DMS-9988110 (D. J. Benson) and DMS-0070776 (N. C. Phillips). 
we consider here, of lifting finite order automorphisms of the K-theory to automorphisms of the algebra of the same order, is a special case of what one gets by restricting the morphisms to be isomorphisms.

Our method is to find, for given countable abelian groups $G_{i}$ and order two automorphisms $\gamma_{i}$, some separable nuclear $\mathrm{C}^{*}$-algebra $A_{0}$ whose $\mathrm{K}$-theory is $G_{*}$ and some order two automorphism $\alpha_{0}$ of $A_{0}$ which lifts the $\gamma_{i}$. In fact, $A_{0}$ will be type I. Then we apply the construction of 15 to get a Kirchberg algebra with the same properties. To construct $A_{0}$, we regard $G_{*}$ as a module over the group ring $\mathbb{Z}\left[\mathbb{Z}_{2}\right]$. (Throughout this paper, $\mathbb{Z}_{p}$ denotes $\mathbb{Z} / p \mathbb{Z}$, not the $p$-adic integers.) Generalizing an old result for the finitely generated case (see Lemma 1 of [11]), we prove that every $\mathbb{Z}\left[\mathbb{Z}_{2}\right]$-module which is free as a $\mathbb{Z}$-module has a direct sum decomposition with only three kinds of summands, namely $\mathbb{Z}\left[\mathbb{Z}_{2}\right]$ itself and $\mathbb{Z}$ on which the nontrivial element of $\mathbb{Z}_{2}$ acts either trivially or by multiplication by -1 . (Butler and Kovács have also obtained similar results, by slightly different methods [5]; a more general result is to appear in 4.) We produce $A_{0}$ by combining this structure theorem with part of Schochet's geometric realization technique from [24].

We want to explicitly point out that the $\mathrm{K}$-theory of a $\mathrm{C}^{*}$-algebra $A$ with an action of $\mathbb{Z}_{2}$, regarded as a $\mathbb{Z}\left[\mathbb{Z}_{2}\right]$-module, is not the same as the $\mathbb{Z}_{2}$-equivariant $\mathrm{K}$-theory of $A$, regarded as a module over the representation ring $R\left(\mathbb{Z}_{2}\right) \cong \mathbb{Z}\left[\mathbb{Z}_{2}\right]$. It is, however, the same as the $\mathbb{Z}_{2}$-equivariant $\mathrm{K}$-theory of $C^{*}\left(\mathbb{Z}_{2}, A\right)$, using the dual action. (Compare with Lemma 4.4) Thus, as a corollary of our construction we obtain a realization theorem, Theorem 4.7 for $R\left(\mathbb{Z}_{2}\right)$-modules as the equivariant $\mathrm{K}$-theory of actions of $\mathbb{Z}_{2}$ on Kirchberg algebras. There is also a version for type I $\mathrm{C}^{*}$-algebras.

The first author would like to thank the MSRI for its hospitality while this work was in progress. The second and third authors would like to thank the organizers of the conference "Aperiodic Order, Dynamical Systems, Operator Algebras, and Topology", during which valuable discussions were held. The third author would like to acknowledge the hospitality of the Mathematisches Forschungsinstitut Oberwolfach, where Elliott's raised his question and where Example[5.1 was constructed, and of the University of Georgia for its invitation for a visit in the spring of 2002.

This paper is organized as follows. In the rest of this section, we demonstrate the failure of the classification functor to split. In Section 2 we prove a structure theorem for $\mathbb{Z}\left[\mathbb{Z}_{2}\right]$-modules which are free as $\mathbb{Z}$-modules. In Section 3 we prove the weak form of realization, using some type I $\mathrm{C}^{*}$-algebra with the right K-theory. In Section 4 we prove the main result, and also the realization theorems for $\mathbb{Z}_{2^{-}}$ equivariant K-theory. Section [5 contains an explicit formula for the order two automorphism in the simplest nontrivial case of our theorem, namely the nontrivial automorphism of $K_{*}\left(M_{3}\left(\mathcal{O}_{4}\right)\right)$.

Here is the example which shows that the most obvious sort of splitting of the classification functor is not possible.

Example 1.1. There is no functor $F$, from the category of $\mathbb{Z}_{2}$-graded abelian groups $G_{*}$ with distinguished element $g$ in degree zero and graded homomorphisms preserving the distinguished elements, to the category of unital Kirchberg algebras satisfying the Universal Coefficient Theorem of [23] and unital homomorphisms, such that $K_{*}\left(F\left(G_{*}, g\right)\right) \cong G_{*}$ with $\left[1_{F\left(G_{*}, g\right)}\right] \mapsto g$ for all $G_{*}$ and $g$, and such that 
the diagram

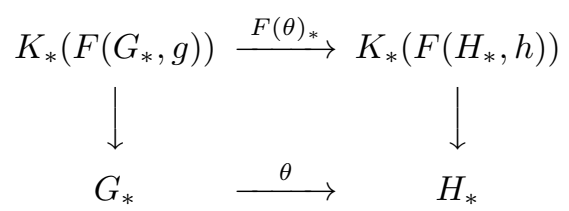

commutes whenever $\theta: G_{*} \rightarrow H_{*}$ is a graded homomorphism such that $\theta(g)=h$.

Suppose we had such a functor. Take

$$
G_{0}=0, \quad G_{1}=0, \quad H_{0}=\mathbb{Z}, \quad \text { and } \quad H_{1}=0 .
$$

Take $g=0$ and $h=0$. Let $\theta: G_{*} \rightarrow H_{*}$ and $\rho: H_{*} \rightarrow G_{*}$ be the unique homomorphisms between these groups. Then $\rho \circ \theta=\mathrm{id}_{G_{*}}$. Therefore, using functoriality,

$$
\left.F(\rho) \circ F(\theta)=F(\rho \circ \theta)=F\left(\operatorname{id}_{G_{*}}\right)=\operatorname{id}_{F\left(G_{*}\right.}\right) .
$$

In particular, $F(\rho)$ is surjective. However, $F\left(G_{*}, g\right)$ (which is isomorphic to the Cuntz algebra $\mathcal{O}_{2}[6]$ ) and $F\left(H_{*}, h\right)$ (which is isomorphic to a suitable corner of $\mathcal{O}_{\infty}$ ) are nonisomorphic simple $\mathrm{C}^{*}$-algebras, so there are no surjective homomorphisms from $F\left(H_{*}, h\right)$ to $F\left(G_{*}, g\right)$.

Remark 1.2. The problem here has nothing to do with units, since the same argument works even if one considers stable Kirchberg algebras satisfying the Universal Coefficient Theorem. Moreover, one can even restrict, in either case, to the case $K_{1}=0$.

\section{Modules OVER The GROUP RING OF $\mathbb{Z}_{2}$}

In this section, we prove a structure theorem for modules over the group ring of $\mathbb{Z}_{2}$ which are free as $\mathbb{Z}$-modules. We have learned that Butler and Kovác proved the main result of this section a little earlier than we did, using slightly different methods [5]. They use a sophisticated argument to reduce to the countably generated case first, which is avoided in our analysis. There is also a subsequent generalization to modules over the group ring of $\mathbb{Z}_{p}$ for an arbitrary prime $p$. We have decided to retain our proof for completeness of exposition, and because it gives the result we need with a minimum of machinery.

For any unital ring $R$ and any discrete group $\Gamma$, we let $R[\Gamma]$ denote the ordinary algebraic group ring of $\Gamma$ with coefficients in $R$. Although we are ultimately interested in the case $R=\mathbb{Z}$, we will need the cases $R=\mathbb{F}_{2}$, the field with two elements, and $R=\mathbb{Q}$. Also, any $\mathbb{Z}[\Gamma]$-module is automatically a $\mathbb{Z}$-module (abelian group) by restriction of scalars.

The structure theorem is already known for the finitely generated case (see the first lemma), and the main point of this section is to remove the finite generation hypothesis.

Lemma 2.1. Let $N$ be a finitely generated $\mathbb{Z}\left[\mathbb{Z}_{2}\right]$-module which is free as a $\mathbb{Z}$ module. Then $N$ is a finite direct $\operatorname{sum} \bigoplus_{i \in I} N_{i}$ in which each $N_{i}$ is isomorphic to one of the following three $\mathbb{Z}\left[\mathbb{Z}_{2}\right]$-modules:

- $T_{1}=\mathbb{Z}$ with the nontrivial element of $\mathbb{Z}_{2}$ acting trivially.

- $T_{2}=\mathbb{Z}$ with the nontrivial element of $\mathbb{Z}_{2}$ acting by multiplication by -1 .

- $T_{3}=\mathbb{Z}\left[\mathbb{Z}_{2}\right]$.

The multiplicities of $T_{1}, T_{2}$ and $T_{3}$ in such a direct sum decomposition are independent of the decomposition. 
Proof. This is an immediate consequence of the canonical form for invertible elements $a \in M_{n}(\mathbb{Z})$ with $a^{2}=1$, given in Lemma 1 of [11, and the discussion following the proof of that lemma. For the matrix $L$ of [11], the computation

$$
L=\left(\begin{array}{cc}
1 & 0 \\
1 & -1
\end{array}\right)=\left(\begin{array}{cc}
1 & -1 \\
0 & 1
\end{array}\right)^{-1}\left(\begin{array}{ll}
0 & 1 \\
1 & 0
\end{array}\right)\left(\begin{array}{cc}
1 & -1 \\
0 & 1
\end{array}\right)
$$

shows that it is similar over $\mathbb{Z}$ to the action of the nontrivial element of $\mathbb{Z}_{2}$ on $\mathbb{Z}\left[\mathbb{Z}_{2}\right]$ in its usual basis.

Theorem 74.3 of [ 8 gives a structure theorem for finitely generated $\mathbb{Z}\left[\mathbb{Z}_{p}\right]$-modules which are free as a $\mathbb{Z}$-modules, for arbitrary primes $p$. As described there, the direct sum decomposition is in general not unique.

Lemma 2.2. Let $M$ be a $\mathbb{Z}\left[\mathbb{Z}_{2}\right]$-module. Suppose $M$ is free as a $\mathbb{Z}$-module and $M / 2 M$ is free as an $\mathbb{F}_{2}\left[\mathbb{Z}_{2}\right]$-module. Then $M$ is free as a $\mathbb{Z}\left[\mathbb{Z}_{2}\right]$-module.

Proof. Theorem 6.1 of [2], with $G=\mathbb{Z}_{2}$ and $H=\{0\}$, shows that $M$ is projective as a $\mathbb{Z}\left[\mathbb{Z}_{2}\right]$-module.

If $M$ is finitely generated, apply Lemma 2.1 Since $T_{1}$ and $T_{2}$ are not projective as $\mathbb{Z}\left[\mathbb{Z}_{2}\right]$-modules, they can't appear in the direct sum. Therefore $M$ is free.

So suppose $M$ is not finitely generated. A direct calculation shows that $\mathbb{Z}\left[\mathbb{Z}_{2}\right]$ has no nontrivial idempotents. (This is true for any finite group in place of $\mathbb{Z}_{2}$, by Corollary 8.1 of [26].) Therefore Corollary 4.5 of [1] applies, and shows that $M$ is free.

Notation 2.3. For the rest of this section, we regard $\mathbb{Z}\left[\mathbb{Z}_{2}\right]$ as a subring of $\mathbb{Q}\left[\mathbb{Z}_{2}\right]$. If $M$ is a $\mathbb{Z}\left[\mathbb{Z}_{2}\right]$-module, then the corresponding $\mathbb{Q}\left[\mathbb{Z}_{2}\right]$-module is $\mathbb{Q} \otimes_{\mathbb{Z}} M$, and if $M$ is free as a $\mathbb{Z}$-module then we may clearly regard $M$ as a submodule of $\mathbb{Q} \otimes_{\mathbb{Z}} M$. We let $s$ be the nontrivial element of $\mathbb{Z}_{2}$, and use the same notation for the corresponding element of $\mathbb{Z}\left[\mathbb{Z}_{2}\right]$. Further set

$$
e=\frac{1}{2}(1+s) \in \mathbb{Q}\left[\mathbb{Z}_{2}\right] \text { and } f=\frac{1}{2}(1-s) \in \mathbb{Q}\left[\mathbb{Z}_{2}\right],
$$

which are idempotents with $e+f=1$.

Lemma 2.4. Let $M$ be a $\mathbb{Z}\left[\mathbb{Z}_{2}\right]$-module which is free as a $\mathbb{Z}$-module. Let $m \in M$, and assume $(1+s) m \in 2 M$. Then, following Notation 2.3

$$
m \in(M \cap e M)+(M \cap f M) \subset \mathbb{Q} \otimes_{\mathbb{Z}} M .
$$

Proof. We have $(1-s) m=(1+s) m-2 m \in 2 M$. Write $(1+s) m=2 m_{0}$ and $(1-s) m=2 m_{1}$ with $m_{0}, m_{1} \in M$. In $\mathbb{Q} \otimes_{\mathbb{Z}} M$ we have $m_{0}=\frac{1}{2}(1+s)=\mathrm{em}$ and $m_{1}=f m$. Then $m_{0} \in M \cap e M$ and $m_{1} \in M \cap f M$. Moreover, $2 m=2\left(m_{0}+m_{1}\right)$, so $m=m_{0}+m_{1}$.

Lemma 2.5. Let $M$ be a $\mathbb{Z}\left[\mathbb{Z}_{2}\right]$-module which is free as a $\mathbb{Z}$-module. Following Notation 2.3 suppose that $M \cap e M=2 e M$ and $M \cap f M=2 f M$. Then $M$ is free as a $\mathbb{Z}\left[\mathbb{Z}_{2}\right]$-module.

Proof. By Lemma 2.2 it suffices to show that $M / 2 M$ is free as an $\mathbb{F}_{2}\left[\mathbb{Z}_{2}\right]$-module.

Set $N=(2 e M+2 f M) / M$. Consider $1+s$ as a multiplication map on $M / 2 M$. We claim that

$$
\operatorname{Ker}(1+s)=(1+s)(M / 2 M)=N
$$


We consider $\operatorname{Ker}(1+s)$ first. Suppose $m \in M$ and $m+2 M \in \operatorname{Ker}(1+s)$. Then $(1+s) m \in 2 M$. Using Lemma 2.4 at the first step and the hypothesis at the second, we get

$$
m \in(M \cap e M)+(M \cap f M)=2 e M+2 f M,
$$

so $m+2 M \in N$. For the reverse, suppose $m \in 2 e M+2 f M$. Write

$$
m=(1+s) m_{0}+(1-s) m_{1}
$$

with $m_{0}, m_{1} \in M$. Then

$$
(1+s) m=(1+s)^{2} m_{0}+(1+s)(1-s) m_{1}=2(1+s) m_{0} \in 2 M,
$$

so $m+2 M \in \operatorname{Ker}(1+s)$.

Now we consider the range of $1+s$. Since $(1+s)^{2}=0$ in $\mathbb{F}_{2}\left[\mathbb{Z}_{2}\right]$, we get $(1+s)(M / 2 M) \subset \operatorname{Ker}(1+s)=N$. For the reverse, let $m \in 2 e M+2 f M$, and again write $m=(1+s) m_{0}+(1-s) m_{1}$ with $m_{0}, m_{1} \in M$. Then

$$
\begin{aligned}
m+2 M=(1+s)\left(m_{0}+m_{1}\right)-2 s m_{1}+2 M & =(1+s)\left(m_{0}+m_{1}\right)+2 M \\
& \in(1+s)(M / 2 M) .
\end{aligned}
$$

This completes the proof of the claim.

Now $N$ is a $\mathbb{F}_{2}$-vector subspace of $M / 2 M$, so there exists a $\mathbb{F}_{2}$-vector subspace $V \subset M / 2 M$ such that $V \oplus N=M / 2 M$. We claim that $V \cap s V=\{0\}$ and $V+s V=M / 2 M$. To prove the first, suppose $v, w \in V$ and $v=s w$. Then $(1+s) v=(1+s) s w=(1+s) w$, so that $(1+s)(v-w)=0$. Therefore $v-w \in$ $\operatorname{Ker}(1+s) \cap V=N \cap V$, whence $v=w$. Now $(1+s) v=v+w=2 v=0$, so $v \in(1+s)(M / 2 M) \cap V=N \cap V$, whence $v=0$. For the second, let $d \in M / 2 M$. Using the first claim, we have $N=(1+s)(M / 2 M)=(1+s)(V)$. So we can write $d=x+(1+s) w$ with $w, x \in V$. Then $d=v+s w$ with $v=x+s w$ and $w$ both in $V$. This proves the claim.

Given the last claim, it is immediate that $M / 2 M \cong \mathbb{F}_{2}\left[\mathbb{Z}_{2}\right] \otimes_{\mathbb{F}_{2}} V$, which is a free $\mathbb{F}_{2}\left[\mathbb{Z}_{2}\right]$-module.

We only need the next two lemmas for the prime 2. Since they hold for arbitrary primes, with the same proof, we may as well give them in that generality.

Lemma 2.6. Let $M$ be a free $\mathbb{Z}$-module, and let $p$ be a prime number. Let $V$ be an $\mathbb{F}_{p}$-vector subspace of $M / p M$. Then there exists a direct summand $L$ of $M$ such that the image of $L$ under the map $M \rightarrow M / p M$ is exactly $V$.

Proof. Let $\pi: M \rightarrow M / p M$ be the quotient map. Choose an $\mathbb{F}_{p}$-vector subspace $W$ of $M / p M$ such that $M / p M=V \oplus W$. Let $\sigma: M / p M \rightarrow W$ be the projection onto $W$ obtained from this direct sum decomposition. Using an $\mathbb{F}_{p}$-basis for $W$, find a free $\mathbb{Z}$-module $P_{0}$ with an isomorphism $P_{0} / p P_{0} \rightarrow W$. Let $\kappa_{0}: P_{0} \rightarrow W$ be the composite of this isomorphism with the quotient map $P_{0} \rightarrow P_{0} / p P_{0}$. Since $M$ is a projective $\mathbb{Z}$-module and $\kappa_{0}$ is surjective, there exists a $\mathbb{Z}$-module homomorphism $\varphi_{0}: M \rightarrow P_{0}$ making the following diagram commute:

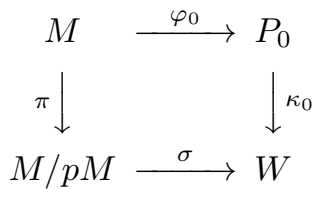


The image $\varphi_{0}(M)$ is a submodule of a free $\mathbb{Z}$-module, hence free (Theorem 14.5 of [10]). Let $\left(b_{i}\right)_{i \in I_{0}}$ be a $\mathbb{Z}$-basis for $\varphi_{0}(M)$. Since $\kappa_{0} \circ \varphi_{0}=\sigma \circ \pi$ is surjective, $\left\{\kappa_{0}\left(b_{i}\right): i \in I_{0}\right\}$ spans $W$. Choose a subset $I \subset I_{0}$ such that $\left(\kappa_{0}\left(b_{i}\right)\right)_{i \in I}$ is an $\mathbb{F}_{p}$-basis for $W$. Set $P=\operatorname{span}_{\mathbb{Z}}\left(\left\{b_{i}: i \in I\right\}\right) \subset P_{0}$, which is a free $\mathbb{Z}$-module with basis $\left(b_{i}\right)_{i \in I}$. For each $j \in I_{0} \backslash I$, choose $\alpha_{i, j} \in \mathbb{F}_{p}$ for $i \in I$, all but finitely many of which are zero, such that $\kappa_{0}\left(b_{j}\right)=\sum_{i \in I} \alpha_{i, j} \kappa_{0}\left(b_{i}\right)$. For $j \in I_{0} \backslash I$ and $i \in I$, choose $\beta_{i, j} \in \mathbb{Z}$ whose image in $\mathbb{F}_{p}$ is $\alpha_{i, j}$, and such that if $\alpha_{i, j}=0$ then $\beta_{i, j}=0$. For each $j \in I_{0} \backslash I$, all but finitely many of the $\beta_{i, j}$ are zero. Therefore there is a well defined surjective $\mathbb{Z}$-module homomorphism $\mu: \varphi_{0}(M) \rightarrow P$ such that $p\left(b_{j}\right)=b_{j}$ for $j \in I$ and $\mu\left(b_{j}\right)=\sum_{i \in I} \beta_{i, j} b_{i}$ for $j \in I_{0} \backslash I$. By construction, we have $\kappa_{0} \circ \mu\left(b_{i}\right)=\kappa_{0}\left(b_{i}\right)$ for all $i \in I$. Therefore $\left(\left.\kappa_{0}\right|_{P}\right) \circ \mu=\kappa_{0}$. Setting $\varphi=\mu \circ \varphi_{0}$ and $\kappa=\left.\kappa_{0}\right|_{P}$, we thus have a commutative diagram:

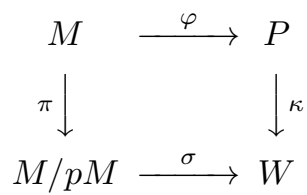

The $\mathbb{Z}$-module $P$ is still free, and in addition $\varphi$ is surjective. Furthermore, $\operatorname{Ker}(\kappa)=$ $p P$, since a linear combination of $\left(b_{i}\right)_{i \in I}$ has image in $W$ equal to zero if and only if all coefficients are even.

Set $L=\operatorname{Ker}(\varphi)$. Since $P$ is projective, there is a $\mathbb{Z}$-module homomorphism $\eta: P \rightarrow M$ such that $\varphi \circ \eta=\operatorname{id}_{P}$. Then $M=L \oplus \eta(P)$. We claim that $\pi(L)=V$. First, if $m \in L$ then $\sigma(\pi(m))=\kappa(\varphi(m))=0$, so $\pi(m) \in \operatorname{Ker}(\sigma)=V$. Now suppose $v \in V$. Choose $m_{0} \in M$ such that $\pi\left(m_{0}\right)=v$. Then $\kappa\left(\varphi\left(m_{0}\right)\right)=0$, whence $\varphi\left(m_{0}\right) \in p P$. Write $\varphi\left(m_{0}\right)=p x$ with $x \in P$. Then $m=m_{0}-p \eta(x) \in \operatorname{Ker}(\varphi)$ by a diagram chase, and $\pi(m)=\pi\left(m_{0}\right)=v$. This proves the claim, and the lemma.

Lemma 2.7. Let $M$ be a free $\mathbb{Z}$-module and let $p$ be a prime number. Let $N$ be a $\mathbb{Z}$ submodule such that $p M \subset N \subset M$. Then there exist $\mathbb{Z}$-submodules $N_{0}, N_{1} \subset M$ such that $M=N_{0} \oplus N_{1}$ and $N=p N_{0} \oplus N_{1}$.

Proof. Let $\pi: M \rightarrow M / p M$ be the quotient map. Using Lemma 2.6] write $M=$ $N_{0} \oplus N_{1}$ for $\mathbb{Z}$-submodules $N_{0}, N_{1} \subset M$, with $\pi\left(N_{1}\right)=\pi(N)$. The result will now follow if we prove that $N=p N_{0}+N_{1}$.

We prove $p N_{0}+N_{1} \subset N$. Clearly $p N_{0} \subset N$. So let $m \in N_{1}$. Choose $n \in N$ such that $\pi(n)=\pi(m)$. Then $\pi(m-n)=0$, so $m-n \in p M \subset N$. Thus $m \in N$. We have $N_{1} \subset N$.

Now we prove the reverse inclusion. Let $n \in N$. Write $n=r+m_{1}$ with $r \in N_{0}$ and $m_{1} \in N_{1}$. Then $\pi(r)=\pi(n)-\pi\left(m_{1}\right)$. Because $N_{1} \subset N$, we get $\pi(r) \in \pi\left(N_{0}\right) \cap \pi(N)$. By considering a $\mathbb{Z}$-basis for $M$ made up of bases for $N_{0}$ and $N_{1}$, we see that $\pi\left(N_{0}\right) \cap \pi(N)=\{0\}$. Therefore $\pi(r)=0$. So we can write $r=p m_{0}$ with $m_{0} \in M$. Since $M$ is torsion free and $N_{0}$ is a summand, we get $m_{0} \in N_{0}$. Therefore $n=p m_{0}+m_{1} \in p N_{0}+N_{1}$.

The uniqueness statement in the following theorem will not be used in the rest of the paper, but we include it because it seems of independent interest.

Theorem 2.8. Let $M$ be a $\mathbb{Z}\left[\mathbb{Z}_{2}\right]$-module which is free as a $\mathbb{Z}$-module. Then $M$ is a direct sum $\bigoplus_{i \in I} M_{i}$ in which each $M_{i}$ is isomorphic to one of the three modules $T_{1}, T_{2}$, or $T_{3}$ of Lemma 2.1. The multiplicities of $T_{1}, T_{2}$ and $T_{3}$ in such a direct sum decomposition are independent of the decomposition. 
Proof. We use Notation 2.3

We first prove uniqueness of the multiplicities. Suppose the $\mathbb{Z}\left[\mathbb{Z}_{2}\right]$-module $M$ is isomorphic to the direct sum of $n_{1}$ copies of $T_{1}, n_{2}$ copies of $T_{2}$, and $n_{3}$ copies of $T_{3}$. Then $n_{1}$ is the $\mathbb{F}_{2}$-vector space dimension of $(M \cap e M) / 2 e M \subset e M / 2 e M$, and similarly $n_{2}=\operatorname{dim}_{\mathbb{F}_{2}}((M \cap f M) / 2 f M)$. Finally, one checks that $n_{3}=\operatorname{dim}_{\mathbb{F}_{2}}(e M /(M \cap$ $e M))$, which is the same as $\operatorname{dim}_{\mathbb{F}_{2}}(f M /(M \cap f M))$.

Now we prove existence. We have

$$
2 e M \subset M \cap e M \subset e M \text { and } 2 f M \subset M \cap f M \subset f M .
$$

Apply Lemma 2.7 twice, to get $\mathbb{Z}$-submodules $R_{0}, R_{1} \subset e M$ and $S_{0}, S_{1} \subset f M$ such that

$$
e M=R_{0} \oplus R_{1} \quad \text { and } \quad M \cap e M=2 R_{0} \oplus R_{1}
$$

and

$$
f M=S_{0} \oplus S_{1} \quad \text { and } \quad M \cap f M=2 S_{0} \oplus S_{1} .
$$

Since $e M \cap f M=\{0\}$ and $e+f=1$, we can write

$$
(M \cap e M) \oplus(M \cap f M) \subset M \subset e M \oplus f M .
$$

Substituting from above, we have the inclusions of $\mathbb{Z}$-module direct sums

$$
2 R_{0} \oplus R_{1} \oplus 2 S_{0} \oplus S_{1} \subset M \subset R_{0} \oplus R_{1} \oplus S_{0} \oplus S_{1} .
$$

Since $R_{1}, S_{1} \subset M$, we have the $\mathbb{Z}$-module direct sum decomposition

$$
M=R_{1} \oplus S_{1} \oplus N \quad \text { with } \quad N=M \cap\left(R_{0} \oplus S_{0}\right) .
$$

Now $R_{1} \subset e M$ and $s m=m$ for all $m \in e M$. So $R_{1}$ is actually a $\mathbb{Z}\left[\mathbb{Z}_{2}\right]$-module. Since it is a $\mathbb{Z}$-submodule of $M$, it is a free $\mathbb{Z}$-module (Theorem 14.5 of [10]), and therefore as a $\mathbb{Z}\left[\mathbb{Z}_{2}\right]$-module it is a direct sum of $\mathbb{Z}\left[\mathbb{Z}_{2}\right]$-modules isomorphic to $T_{1}$. Similarly, from $S_{1} \subset f M$ and $s m=-m$ for all $m \in f M$, we see that $S_{1}$ is a $\mathbb{Z}\left[\mathbb{Z}_{2}\right]$-module which is a direct sum of $\mathbb{Z}\left[\mathbb{Z}_{2}\right]$-modules isomorphic to $T_{2}$. We will complete the proof by showing that $N$ is a $\mathbb{Z}\left[\mathbb{Z}_{2}\right]$-module, so that the direct sum decomposition above is really a $\mathbb{Z}\left[\mathbb{Z}_{2}\right]$-module direct sum decomposition, and that it is free, so that it is a direct sum of $\mathbb{Z}\left[\mathbb{Z}_{2}\right]$-modules isomorphic to $T_{3}$.

To prove that $N$ is a $\mathbb{Z}\left[\mathbb{Z}_{2}\right]$-module, we simply observe that $R_{0}$ and $S_{0}$ are $\mathbb{Z}\left[\mathbb{Z}_{2}\right]$ modules, for the same reason that $R_{1}$ and $S_{1}$ are. For freeness, we know as above that $N$ is a free $\mathbb{Z}$-module because it is a $\mathbb{Z}$-submodule of $M$. By Lemma 2.5 it now suffices to prove that $N \cap e N=2 e N$ and $N \cap f N=2 f N$. That $2 e N \subset N \cap e N$ and $2 f N \subset N \cap f N$ is clear, so we prove the reverse inclusions.

Let $m \in N \cap e N$. Use $m \in N$ to write $m=a+b$ with $a \in R_{0}$ and $b \in S_{0}$. Use $m \in e N$ to write $m=e(x+y)$ with $x \in R_{0}$ and $y \in S_{0}$. Note that $e x=x$ and $e y=0$, and combine the two expressions for $m$ to get $x-a=b$. Since $x-a \in e M$ and $b \in f M$, we get $b=0$. Therefore $m=a \in R_{0}$. From $m \in M \cap e M=2 R_{0} \oplus R_{1}$ we get $m \in 2 R_{0}$. Now $R_{0} \subset e M$ implies $e R_{0}=R_{0}$, so $R_{0} \subset N$ implies $R_{0} \subset e N$. Therefore $m \in 2 e N$, as desired.

Now suppose $m \in N \cap f N$. Write $m=a+b=f(x+y)$ with $a, x \in R_{0}$ and $b, y \in S_{0}$. From $f x=0$ and $f y=y$, we get $y-b=a \in e M \cap f M=\{0\}$. So $m=b \in S_{0}$. From $m \in M \cap f M=2 S_{0} \oplus S_{1}$ we now get $m \in 2 S_{0}$. Since $S_{0} \subset N \cap f M \subset f N$, it follows that $m \in 2 f N$, as desired. This completes the proof. 


\section{Geometric Resolution}

In this section, we prove that for every order two automorphism $\gamma$ of a countable $\mathbb{Z}_{2}$-graded abelian group $G_{*}$, there is some separable $\mathrm{C}^{*}$-algebra $A$ with an order two automorphism $\alpha \in \operatorname{Aut}(A)$, such that there is a graded isomorphism $\mu: G_{*} \rightarrow$ $K_{*}(A)$ which identifies $\gamma$ with $\alpha_{*}$. We borrow the method of geometric realization of resolutions from Schochet's proof of the Künneth formula for $\mathrm{C}^{*}$-algebras [24]. As it turns out, the $\mathrm{C}^{*}$-algebra $A$ we produce will be of type I and nonunital.

Although we do not state this version formally, the same proofs show that if $G_{*}$ is not countable, we can still produce an automorphism of a nonseparable type I $\mathrm{C}^{*}$-algebra satisfying the remaining conditions.

For any $\mathrm{C}^{*}$-algebra $A$, we let $A^{+}$denote its usual unitization, in which we add a new unit even if $A$ is already unital.

Lemma 3.1. Let $C=\bigoplus_{n \in \mathbb{N}}\left[C_{0}(\mathbb{R}) \oplus C_{0}(\mathbb{R})\right]$, and let $\gamma \in \operatorname{Aut}(C)$ be the order two automorphism given by

$$
\gamma\left(\left(f_{1}, g_{1}\right),\left(f_{2}, g_{2}\right), \ldots\right)=\left(\left(g_{1}, f_{1}\right),\left(g_{2}, f_{2}\right), \ldots\right) .
$$

Let $M$ be a subgroup of $K_{1}(C)$ which is invariant under $\gamma_{*}$. Then there exists a separable Hilbert space $H$ with an action of $\mathbb{Z}_{2}$, a separable commutative $\mathrm{C}^{*}$-algebra $B$ with an automorphism $\beta$ of order two, and a $\mathbb{Z}_{2}$-equivariant homomorphism $\varphi: B \rightarrow K(H) \otimes C$, where $\mathbb{Z}_{2}$ acts on $K(H)$ by conjugation, such that $K_{0}(B)=0$, such that $\varphi_{*}: K_{1}(B) \rightarrow K_{1}(C)$ is injective, and such that the image of $\varphi_{*}$ is exactly $M$.

Proof. The K-theory $K_{*}(C)$ is a countable abelian group with an automorphism $\gamma_{*}$ of order two, and hence in an obvious way a $\mathbb{Z}\left[\mathbb{Z}_{2}\right]$-module. As $\mathbb{Z}\left[\mathbb{Z}_{2}\right]$-modules, $K_{1}(C)$ is a countable direct sum of copies of $\mathbb{Z}\left[\mathbb{Z}_{2}\right]$ and $K_{0}(C)=0$. Moreover, the hypothesis on $M$ is just that it is a $\mathbb{Z}\left[\mathbb{Z}_{2}\right]$-submodule. It is a free $\mathbb{Z}$-module because subgroups of free abelian groups are free (Theorem 14.5 of [10]), and it is clearly countably generated, so by Theorem 2.8 there is an isomorphism $M \cong \bigoplus_{i \in I} M_{i}$ with $I$ finite or countable, and with each $M_{i}$ isomorphic to one of the three modules $T_{j}$ of Lemma 2.1] We regard each $M_{i}$ as a $\mathbb{Z}\left[\mathbb{Z}_{2}\right]$-submodule of $K_{1}(C)$.

For each $i \in I$, we construct a separable Hilbert space $H_{i}$ with an action of $\mathbb{Z}_{2}$, a separable commutative $\mathrm{C}^{*}$-algebra $B_{i}$ with an automorphism $\beta_{i}$ of order two, and a $\mathbb{Z}_{2}$-equivariant homomorphism $\varphi_{i}: B_{i} \rightarrow K\left(H_{i}\right) \otimes C$, such that $K_{0}\left(B_{i}\right)=0$, such that $\left(\varphi_{i}\right)_{*}: K_{1}\left(B_{i}\right) \rightarrow K_{1}(C)$ is injective, and such that the image of $\left(\varphi_{i}\right)_{*}$ is exactly $M_{i}$. There are three cases, but we begin by introducing notation common to all three. Define $u: \mathbb{R} \rightarrow S^{1}$ by

$$
u(t)=\exp \left(\pi i\left(1+t\left(1+t^{2}\right)^{-1 / 2}\right)\right) .
$$

Then $u$ is a unitary in the unitization $C_{0}(\mathbb{R})^{+}$such that $[u]$ generates $K_{1}\left(C_{0}(\mathbb{R})\right)$, and $u-1$ generates $C_{0}(\mathbb{R})$ as a $\mathrm{C}^{*}$-algebra. In particular, if $E$ is any $\mathrm{C}^{*}$-algebra and $v \in E^{+}$is any unitary such that $v-1 \in E$, then there is a unique homomorphism $\psi: C_{0}(\mathbb{R}) \rightarrow E$ such that $\psi(u-1)=v-1$. Further let $s$ be the nontrivial element of $\mathbb{Z}_{2}$, and use the same notation for the corresponding element of $\mathbb{Z}\left[\mathbb{Z}_{2}\right]$. We will also write elements of $K_{1}(C)$ as sequences

$$
\left(k_{1}+l_{1} s, k_{2}+l_{2} s, \ldots\right) \in \bigoplus_{n \in \mathbb{N}} \mathbb{Z}\left[\mathbb{Z}_{2}\right]
$$


where, with all nontrivial entries being in the $n$-th position, $(0, \ldots, 0,1,0, \ldots)$ corresponds to the $K_{1}$-class of the unitary

$$
1+((0,0), \ldots,(0,0),(u-1,0),(0,0), \ldots) \in C^{+}
$$

and $(0, \ldots, 0, s, 0, \ldots)$ corresponds to the $K_{1}$-class of the unitary

$$
1+((0,0), \ldots,(0,0),(0, u-1),(0,0), \ldots) \in C^{+} .
$$

The first case is $M_{i} \cong T_{1}$. Let $m$ be a generator of $M_{i}$ as a $\mathbb{Z}$-module. Then $s m=m$. Therefore $m$ has the form

$$
m=\left(k_{1}+k_{1} s, k_{2}+k_{2} s, \ldots\right),
$$

with all but finitely many of the $k_{j}$ equal to zero. Take $H_{i}=\mathbb{C}$ with the trivial action of $\mathbb{Z}_{2}$, take $B_{i}=C_{0}(\mathbb{R})$, take $\beta_{i}=\operatorname{id}_{B_{i}}$, and take $\varphi_{i}$ to be the homomorphism determined by

$$
\varphi_{i}(u-1)=\left(\left(u^{k_{1}}-1, u^{k_{1}}-1\right),\left(u^{k_{2}}-1, u^{k_{2}}-1\right), \ldots\right) .
$$

The required properties are immediate.

Next, suppose $M_{i} \cong T_{2}$. Let $m$ be a generator of $M_{i}$ as a $\mathbb{Z}$-module. Then $s m=-m$. Therefore $m$ has the form

$$
m=\left(k_{1}-k_{1} s, k_{2}-k_{2} s, \ldots\right),
$$

with all but finitely many of the $k_{j}$ equal to zero. Take $H_{i}=\mathbb{C}$ with the trivial action of $\mathbb{Z}_{2}$, and take $B_{i}=C_{0}(\mathbb{R})$. Take $\beta_{i}: C_{0}(\mathbb{R}) \rightarrow C_{0}(\mathbb{R})$ to be the homomorphism $\beta_{i}(f)(t)=f(-t)$, which is the unique homomorphism such that $\beta_{i}(u-1)=u^{*}-1$. Take $\varphi_{i}$ to be the homomorphism determined by

$$
\varphi_{i}(u-1)=\left(\left(u^{k_{1}}-1, u^{-k_{1}}-1\right),\left(u^{k_{2}}-1, u^{-k_{2}}-1\right), \ldots\right) .
$$

Equivariance follows from

$$
\varphi_{i}\left(u^{*}-1\right)=\left(\left(u^{-k_{1}}-1, u^{k_{1}}-1\right),\left(u^{-k_{2}}-1, u^{k_{2}}-1\right), \ldots\right),
$$

and the rest of the required properties are immediate.

Finally, suppose $M_{i} \cong T_{3}$. Let $m \in K_{1}(C)$ be the image of $1 \in T_{3}$ under the isomorphism $T_{3} \rightarrow M_{i}$. Write $m=\left(k_{1}+l_{1} s, k_{2}+l_{2} s, \ldots\right)$. Then the image of $s$ is

$$
s m=\left(l_{1}+k_{1} s, l_{2}+k_{2} s, \ldots\right),
$$

and these two elements are linearly independent over $\mathbb{Z}$. Take $H_{i}=\mathbb{C}^{2}$, with $s\left(\xi_{1}, \xi_{2}\right)=\left(\xi_{2}, \xi_{1}\right)$. Take $B_{i}=C_{0}(\mathbb{R}) \oplus C_{0}(\mathbb{R})$, with $\beta_{i}\left(f_{1}, f_{2}\right)=\left(f_{2}, f_{1}\right)$. To define

$$
\varphi_{i}: C_{0}(\mathbb{R}) \oplus C_{0}(\mathbb{R}) \rightarrow L\left(\mathbb{C}^{2}\right) \otimes C,
$$

let $p_{1}, p_{2} \in L\left(\mathbb{C}^{2}\right)$ be the projections on the first and second coordinates, and let $\psi_{1}, \psi_{2}: C_{0}(\mathbb{R}) \rightarrow C$ be the unique homomorphisms such that

$$
\psi_{1}(u-1)=\left(\left(u^{k_{1}}-1, u^{l_{1}}-1\right),\left(u^{k_{2}}-1, u^{l_{2}}-1\right), \ldots\right)
$$

and

$$
\psi_{2}(u-1)=\left(\left(u^{l_{1}}-1, u^{k_{1}}-1\right),\left(u^{l_{2}}-1, u^{k_{2}}-1\right), \ldots\right) .
$$

Then define

$$
\varphi_{i}\left(f_{1}, f_{2}\right)=p_{1} \otimes \psi_{1}\left(f_{1}\right)+p_{2} \otimes \psi_{2}\left(f_{2}\right) .
$$

This is a homomorphism because $p_{1}$ and $p_{2}$ are orthogonal. Equivariance follows from the fact that the action of $s$ exchanges $p_{1}$ and $p_{2}$, and the formulas for $m$ and $s m$. That $\left(\psi_{i}\right)_{*}$ is injective follows from the fact that $m$ and $s m$ are linearly independent over $\mathbb{Z}$, and the remaining required properties are clear. 
Now let $H$ be the Hilbert direct sum $H=\bigoplus_{i \in I} H_{i}$, and let $B$ be the $\mathrm{C}^{*}$-algebra direct sum $B=\bigoplus_{i \in I} B_{i}$. Give both the action of $\mathbb{Z}_{2}$ coming from the actions on the summands. Define $\varphi: B \rightarrow K(H) \otimes C$ by taking $\varphi\left(\left(b_{i}\right)_{i \in I}\right)$ to be the block diagonal element $\bigoplus_{i \in I} \varphi_{i}\left(b_{i}\right)$. This element is in fact in $K(H) \otimes C$ because for every $\varepsilon>0$, we have $\left\|b_{i}\right\|<\varepsilon$ for all but finitely many $i$. Then $\varphi$ is the required homomorphism.

Lemma 3.2. Let $G$ be a countable abelian group, and let $\nu: G \rightarrow G$ be an automorphism of $G$ of order two. Then there exist a separable type I $\mathrm{C}^{*}$-algebra $A$ such that $K_{1}(A)=0$, an automorphism $\alpha \in \operatorname{Aut}(A)$ of order two, and an isomorphism $\mu: G \rightarrow K_{0}(A)$, such that $\mu \circ \nu=\alpha_{*} \circ \mu$.

Proof. The group $G$ is a $\mathbb{Z}\left[\mathbb{Z}_{2}\right]$-module in an obvious way, and it is countable by assumption. Set $N=\bigoplus_{n \in \mathbb{N}} \mathbb{Z}\left[\mathbb{Z}_{2}\right]$, and choose a surjective $\mathbb{Z}\left[\mathbb{Z}_{2}\right]$-module homomorphism $\tau: N \rightarrow G$. Set $M=\operatorname{Ker}(\tau)$. Let $C$ be as in Lemma 3.1 and identify $N$ with $K_{1}(C)$. Apply Lemma 3.1] with this $M$, obtaining a $\mathbb{Z}_{2}$-equivariant homomorphism of separable type I $\mathrm{C}^{*}$-algebras $\varphi: B \rightarrow K(H) \otimes C$ such that (by abuse of notation) $K_{1}(B)=M, K_{1}(K(H) \otimes C)=N$, and $\varphi_{*}$ is the inclusion. To simplify the notation, set $D=K(H) \otimes C$.

Let $A$ be the mapping cone

$$
A=\{(f, b) \in C([0,1], D) \oplus B: f(0)=0 \text { and } f(1)=\varphi(b)\} .
$$

Since $\varphi$ is equivariant, this algebra has an obvious action of $\mathbb{Z}_{2}$. (The action is trivial on $[0,1]$.) Moreover, with $S D=C_{0}((0,1), D)$ being the usual suspension of $D$, there is an equivariant short exact sequence

$$
0 \longrightarrow S D \longrightarrow A \longrightarrow B \longrightarrow 0 .
$$

By naturality the corresponding six term exact sequence in $\mathrm{K}$-theory is actually a sequence of $\mathbb{Z}\left[\mathbb{Z}_{2}\right]$-modules. Since $K_{0}(B)=0$ and $K_{0}(D)=0$, this sequence reduces to

$$
0 \longrightarrow K_{1}(A) \longrightarrow K_{1}(B) \longrightarrow K_{0}(S D) \longrightarrow K_{0}(A) \longrightarrow 0 .
$$

Using the Bott periodicity isomorphism $K_{0}(S D) \cong K_{1}(D)$, and appropriately identifying the maps following Theorem 3.5 of [25], this sequence can be naturally identified with

$$
0 \longrightarrow K_{1}(A) \longrightarrow K_{1}(B) \stackrel{\varphi_{*}}{\longrightarrow} K_{1}(D) \longrightarrow K_{0}(A) \longrightarrow 0 .
$$

By our construction and by naturality of the sequence, we therefore have the sequence of $\mathbb{Z}\left[\mathbb{Z}_{2}\right]$-modules

$$
0 \longrightarrow K_{1}(A) \longrightarrow M \stackrel{\tau}{\longrightarrow} N \longrightarrow K_{0}(A) \longrightarrow 0,
$$

from which it follows that $K_{1}(A)=0$ and that $K_{0}(A) \cong N / M \cong G$ as $\mathbb{Z}\left[\mathbb{Z}_{2}\right]$ modules.

Proposition 3.3. Let $G_{0}$ and $G_{1}$ be countable abelian groups. Let $\gamma_{i}$ be an automorphism of $G_{i}$ of order two. Then there exist a separable type I $\mathrm{C}^{*}$-algebra $A$, an automorphism $\alpha \in \operatorname{Aut}(A)$ of order two, and a graded isomorphism $\mu: G_{*} \rightarrow$ 
$K_{*}(A)$, such that the diagram

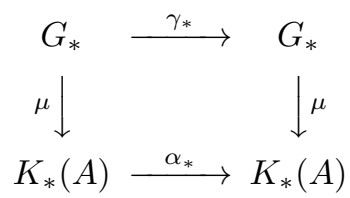

commutes.

Proof. Apply Lemma 3.2 to $G_{0}$ and $\gamma_{0}$, obtaining a $\mathrm{C}^{*}$-algebra $A_{0}$ with an order two automorphism $\alpha_{0}$. Apply Lemma 3.2 to $G_{1}$ and $\gamma_{1}$, obtaining a $\mathrm{C}^{*}$-algebra $A_{1}$ with an order two automorphism $\alpha_{1}$. Take $A=A_{0} \oplus S A_{1}$ and $\alpha=\alpha_{0} \oplus S \alpha_{1}$. This $\mathrm{C}^{*}$-algebra and automorphism satisfy the conclusions by Bott periodicity.

\section{From type I to PURELY infinite Simple}

For countable abelian groups $G_{0}$ and $G_{1}$ and order two automorphisms $\gamma_{i}$ of $G_{i}$, we can now produce a separable type I C*-algebra $A$ with K-theory $G_{0} \oplus G_{1}$, and an order two automorphism which induces $\gamma_{i}$ on K-theory. We want to use the construction of [15] to produce a unital Kirchberg algebra. However, $A$ is not unital, and this construction will then not produce a unital $\mathrm{C}^{*}$-algebra. Unitizing $A$ changes the K-theory. To remedy this problem, we introduce in the next proposition a kind of unitization functor which does not change the K-theory. Our functor preserves nuclearity and the Universal Coefficient Theorem, but not type I (or simplicity or finiteness). Although we will not use this fact, it also seems interesting to observe that the functor sends exact $\mathrm{C}^{*}$-algebras to exact $\mathrm{C}^{*}$-algebras .

Proposition 4.1. There is a functor $F$ from the category of all $\mathrm{C}^{*}$-algebras and $\mathrm{C}^{*}$ algebra homomorphisms to the subcategory of all unital $\mathrm{C}^{*}$-algebras and unital $\mathrm{C}^{*}$ algebra homomorphisms, and a natural transformation $\eta$ from the identity functor to $F$, such that:

(1) For every $\mathrm{C}^{*}$-algebra $A$, the homomorphism $\eta_{A}: A \rightarrow F(A)$ is a KKequivalence, and in particular is an isomorphism on K-theory.

(2) If $A$ is separable, or nuclear, or exact, or satisfies the Universal Coefficient Theorem of [23, then $F(A)$ also has the same property.

Proof. Choose and fix a nonzero projection $e \in \mathcal{O}_{\infty}$ such that $[e]=0$ in $K_{0}\left(\mathcal{O}_{\infty}\right)$, a projection $q \leq e$ such that $[q]=\left[1_{\mathcal{O}_{\infty}}\right]$ in $K_{0}\left(\mathcal{O}_{\infty}\right)$, and a unital subalgebra $D \subset e \mathcal{O}_{\infty} e$ such that $D \cong \mathcal{O}_{2}$. For any $\mathrm{C}^{*}$-algebra $A$, let $A^{+}$be the unitization (as in Section 3), let $\pi_{A}: A^{+} \rightarrow \mathbb{C}$ be the canonical map, and define

$$
F(A)=\left\{b \in e \mathcal{O}_{\infty} e \otimes A^{+}:\left(\operatorname{id}_{e \mathcal{O}_{\infty} e} \otimes \pi_{A}\right)(b) \in D\right\} .
$$

Let $\iota_{A}: A \rightarrow A^{+}$be the inclusion, and define $\eta_{A}: A \rightarrow F(A)$ by $\eta_{A}(a)=q \otimes \iota_{A}(a)$. This element is in $F(A)$ because $\left(\operatorname{id}_{e \mathcal{O}_{\infty} e} \otimes \pi_{A}\right)\left(q \otimes \iota_{A}(a)\right)=0$.

Since the map $\lambda \mapsto \lambda q$ from $\mathbb{C}$ to $e \mathcal{O}_{\infty} e$ is a KK-equivalence, so is the map $a \mapsto q \otimes a$ from $A$ to $e \mathcal{O}_{\infty} e$. (See Example 19.1.2(c) of [3].) Moreover, there is a split short exact sequence

$$
0 \longrightarrow e \mathcal{O}_{\infty} e \otimes A \longrightarrow F(A) \longrightarrow \mathcal{O}_{2} \longrightarrow 0 .
$$

Since $\mathcal{O}_{2}$ is KK-equivalent to the zero $\mathrm{C}^{*}$-algebra, it follows that, for every separable $\mathrm{C}^{*}$-algebra $B$, the inclusion $\varphi: e \mathcal{O}_{\infty} e \otimes A \rightarrow F(A)$ induces isomorphisms

$$
\varphi_{B}^{*}: K K(F(A), B) \rightarrow K K\left(e \mathcal{O}_{\infty} e \otimes A, B\right)
$$


and

$$
\varphi_{*}^{B}: K K\left(B, e \mathcal{O}_{\infty} e \otimes A\right) \rightarrow K K(B, F(A)) .
$$

It now follows from the Yoneda Lemma that $\varphi$ is a KK-equivalence (see Section III.2 of [16]), but we give the easy direct argument here. In terms of the Kasparov product, these isomorphisms have the formulas $\varphi_{B}^{*}(\gamma)=[\varphi] \cdot \gamma$ and $\varphi_{*}^{B}(\gamma)=\gamma \cdot[\varphi]$. (See Proposition 18.7.2 of [3].) Choose

$$
\alpha \in K K\left(F(A), e \mathcal{O}_{\infty} e \otimes A\right) \quad \text { and } \quad \beta \in K K\left(e \mathcal{O}_{\infty} e \otimes A, F(A)\right)
$$

such that

$$
\varphi_{e \mathcal{O}_{\infty} e \otimes A}^{*}(\alpha)=\left[\operatorname{id}_{e \mathcal{O}_{\infty} e \otimes A}\right] \text { and } \varphi_{*}^{F(A)}(\beta)=\left[\operatorname{id}_{F(A)}\right]
$$

Then

$$
[\varphi] \cdot \alpha=\left[\operatorname{id}_{e \mathcal{O}_{\infty} e \otimes A}\right] \text { and } \beta \cdot[\varphi]=\left[\operatorname{id}_{F(A)}\right],
$$

from which it follows that $\varphi$ is also a KK-equivalence. So $\eta_{A}$ is a KK-equivalence. This proves the property (1).

It is immediate from the KK-equivalence of (1) that $F(A)$ satisfies the Universal Coefficient Theorem if $A$ does. That $F$ preserves separability and nuclearity is immediate from the split exact sequence above, since $e \mathcal{O}_{\infty} e$ and $\mathcal{O}_{2}$ are separable and nuclear. The same argument works for exactness: the minimal tensor product of exact $\mathrm{C}^{*}$-algebras is exact by Proposition 7.1(iii) of [13, and extensions with completely positive splittings preserve exactness by Proposition 7.1(vi) of [13].

Next, we need an equivariant version of the construction of [15].

Proposition 4.2. Let $\alpha: \Gamma \rightarrow \operatorname{Aut}(A)$ be an action of a countable discrete group $\Gamma$ on a separable unital $C^{*}$-algebra $A$. Then there exists a separable unital purely infinite simple $\mathrm{C}^{*}$-algebra $B$, an action $\beta: \Gamma \rightarrow \operatorname{Aut}(B)$, and an equivariant unital homomorphism $\varphi: A \rightarrow B$, such that $\varphi$ is a KK-equivalence. Moreover, if $A$ is nuclear then so is $B$, and if $A$ satisfies the Universal Coefficient Theorem then so does $B$.

Proof. Choose an injective unital representation $\pi_{0}: A \rightarrow L\left(H_{0}\right)$ of $A$ on a separable Hilbert space $H_{0}$ such that $\pi_{0}(A) \cap K\left(H_{0}\right)=\{0\}$. Let $H=l^{2}\left(\Gamma, H_{0}\right)$, and let $u: \Gamma \rightarrow U(H)$ and $\pi: A \rightarrow L(H)$ be the components of the regular covariant representation of $(\Gamma, A)$ associated to $\pi_{0}$, as in 7.7.1 in [17. Then $u_{\gamma} \pi(a) u_{\gamma}^{*}=$ $\pi\left(\alpha_{\gamma}(a)\right)$ for all $a \in A$ and $\gamma \in \Gamma$. (See 17.) Moreover, $\pi(A) \cap K(H)=\{0\}$.

Now we follow the proof of Proposition 2.1 of [15]. Let $E=H \otimes_{\mathbb{C}} A$ be the Hilbert $A$-bimodule defined there, and let $\varphi: A \rightarrow L(E)$ be as there. Each $\gamma \in \Gamma$ induces a isometric $\mathbb{C}$-linear map $v_{\gamma}: E \rightarrow E$ given by $v_{\gamma}(\xi \otimes a)=u_{\gamma} \xi \otimes \alpha_{\gamma}(a)$ for $\xi \in H$ and $a \in A$. These maps have the following properties:

(1) $v_{\gamma}(\xi a)=v_{\gamma}(\xi) \alpha_{\gamma}(a)$ for $\gamma \in \Gamma, \xi \in E$, and $a \in A$.

(2) $\left\langle v_{\gamma} \xi, v_{\gamma} \eta\right\rangle=\alpha_{\gamma}(\langle\xi, \eta\rangle)$ for $\gamma \in \Gamma$ and $\xi, \eta \in E$.

(3) $\left\|v_{\gamma} \xi\right\|=\|\xi\|$ for $\gamma \in \Gamma$ and $\xi \in E$.

(4) $v_{\gamma^{-1}}=v_{\gamma}^{-1}$ for $\gamma \in \Gamma$.

(5) $v_{\gamma} \varphi(a) v_{\gamma}^{-1}=\varphi\left(\alpha_{\gamma}(a)\right)$ for $\gamma \in \Gamma$ and $a \in A$.

Caution: $v_{\gamma}$ is not a right $A$-module homomorphism. Nevertheless, these properties imply that $b \mapsto v_{\gamma} b v_{\gamma}^{-1}$ is a ${ }^{*}$-automorphism of the $\mathrm{C}^{*}$-algebra $L(E)$ of all adjointable right $A$-module morphisms of $E$; moreover, $\varphi$ is equivariant.

Next, let $\mathcal{E}_{+}=\bigoplus_{n=0}^{\infty} E^{\otimes n}$ be the Fock space of $E$, as after Definition 1.3 of [15] or as at the beginning of Section 1 of 20 . We let $\gamma \in \Gamma$ act on $E^{\otimes n}$ via $v_{\gamma}^{\otimes n}$ (via 
$\alpha_{\gamma}$ on $E^{\otimes 0}=A$ ), and on $\mathcal{E}_{+}$via the direct sum $w_{\gamma}$ of these actions. This action is still isometric, and we again have the analogs of Properties (1)-(5) above. The role of $\varphi$ is now played by the homomorphism $\varphi_{+}: A \rightarrow L\left(\mathcal{E}_{+}\right)$of the discussion after Definition 1.3 of 15 . Following the construction there, the Toeplitz algebra $\mathcal{T}_{E}$ is by definition the $\mathrm{C}^{*}$-subalgebra of $L\left(\mathcal{E}_{+}\right)$generated by the operators $T_{\xi}$ defined there, for $\xi \in E$. One checks that $T_{v_{\gamma} \xi}=w_{\gamma} T_{\xi} w_{\gamma}^{-1}$ for $\gamma \in \Gamma$ and $\xi \in E$. Therefore $\Gamma$ acts on $\mathcal{T}_{E}$ via ${ }^{*}$-automorphisms. Since $\pi(A) \cap K(H)=\{0\}$, Lemma 2.1 of [15] and Corollary 3.14 and Theorem 3.13 of $\left[20\right.$ show that the canonical map from $\mathcal{T}_{E}$ to the Cuntz-Pimsner algebra $\mathcal{O}_{E}$ is an isomorphism. Therefore we have an action of $\Gamma$ on $\mathcal{O}_{E}$, and $\varphi_{+}: A \rightarrow \mathcal{O}_{E}$ is equivariant.

Theorem 2.8 of [15] shows that $\mathcal{O}_{E}$ is purely infinite and simple. Separability is clear. The proof of Theorem 3.1 of [15] shows that if $A$ is nuclear, then so is $\mathcal{O}_{E}$. Corollary 4.5 of [20] shows that $\varphi_{+}$is a KK-equivalence, from which it is immediate that if $A$ satisfies the Universal Coefficient Theorem then so does $\mathcal{O}_{E}$.

Theorem 4.3. Let $G_{0}$ and $G_{1}$ be countable abelian groups. Let $\gamma_{i}$ be an automorphism of $G_{i}$ of order two. Then there exist a unital Kirchberg algebra $A$ satisfying the Universal Coefficient Theorem and with $\left[1_{A}\right]=0$ in $K_{0}(A)$, an automorphism $\alpha \in \operatorname{Aut}(A)$ of order two, and a graded isomorphism $\mu: G_{*} \rightarrow K_{*}(A)$, such that the diagram

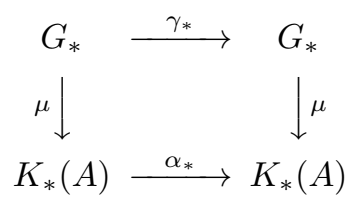

commutes.

Proof. Use Proposition 3.3 to find a separable type I $\mathrm{C}^{*}$-algebra $A_{0}$, an automorphism $\alpha_{0} \in \operatorname{Aut}\left(A_{0}\right)$ of order two, and a graded isomorphism $\mu_{0}: G_{*} \rightarrow K_{*}\left(A_{0}\right)$, such that $\mu_{0} \circ \gamma=\left(\alpha_{0}\right)_{*} \circ \mu_{0}$.

Let $F$ be the functor of Proposition 4.1 and let $\eta: A_{0} \rightarrow F\left(A_{0}\right)$ be the natural transformation from there. By Part (2) of Proposition 4.1 the algebra $F\left(A_{0}\right)$ is separable, unital, nuclear, and satisfies the Universal Coefficient Theorem; also, $\eta$ is a KK-equivalence. Since $F$ is a functor, $F\left(\alpha_{0}\right)$ is an order two automorphism of $F\left(A_{0}\right)$, and naturality implies that $\eta \circ \alpha_{0}=F\left(\alpha_{0}\right) \circ \eta$.

Now use Proposition 4.2 to find a unital Kirchberg algebra $A$ satisfying the Universal Coefficient Theorem, an automorphism $\alpha \in \operatorname{Aut}(A)$ of order two, and a $\mathbb{Z}_{2}$-equivariant homomorphism $\varphi: F\left(A_{0}\right) \rightarrow A$ which is a KK-equivalence. Then $\varphi \circ \eta: A_{0} \rightarrow A$ is $\mathbb{Z}_{2}$-equivariant and is a KK-equivalence. The theorem is therefore proved by taking $\mu=\varphi_{*} \circ \eta_{*} \circ \mu_{0}$.

It should be easy to arrange to have $\left[1_{A}\right]$ correspond to any element in $G$ of the form $\eta+\gamma_{0}(\eta)$, but we don't know how to get arbitrary $\gamma_{0}$-invariant elements of $G_{0}$.

We now turn to the problem of realizing $R\left(\mathbb{Z}_{2}\right)$-modules as equivariant K-theory for actions on $\mathrm{C}^{*}$-algebras. The main point is contained in the next lemma, which we state in greater generality.

Before stating it, recall (Section 2.2 of [18]) that if a compact group $G$ acts on a $\mathrm{C}^{*}$-algebra $A$, then the equivariant $\mathrm{K}$-theory $K_{*}^{G}(A)$ is, in a canonical way, a module over the representation $\operatorname{ring} R(G)$ of $G$. Further recall that when $G$ is 
abelian, the representation ring $R(G)$ is just $\mathbb{Z}[\widehat{G}]$; in particular, if $G$ is a countable abelian group, then $R(\widehat{G})$ is canonically isomorphic to $\mathbb{Z}[G]$.

Lemma 4.4. Let $G$ be a countable abelian group, and let $\alpha: G \rightarrow \operatorname{Aut}(A)$ be an action of $G$ on a $C^{*}$-algebra $A$. Regard $K_{*}(A)$ as a $\mathbb{Z}[G]$-module via the action of $G$ on $A$. Regard the equivariant K-theory $K_{*}^{\widehat{G}}\left(C^{*}(G, A, \alpha)\right)$ of the dual action $\widehat{\alpha}: \widehat{G} \rightarrow$ $\operatorname{Aut}\left(C^{*}(G, A, \alpha)\right)$ as a $\mathbb{Z}[G]$-module as discussed above. Then $K_{*}^{\widehat{G}}\left(C^{*}(G, A, \alpha)\right) \cong$ $K_{*}(A)$ as $\mathbb{Z}[G]$-modules.

Proof. Let $B=C^{*}\left(\widehat{G}, C^{*}(G, A, \alpha), \widehat{\alpha}\right)$ be the second crossed product, with second dual action $\beta: G \rightarrow \operatorname{Aut}(B)$. Regard $K_{*}(B)$ as a $\mathbb{Z}[G]$-module via this action of $G$. Proposition 2.7.10 of [18], applied to the action $\widehat{\alpha}$ of $\widehat{G}$ on $C^{*}(G, A, \alpha)$, shows that $K_{0}^{\widehat{G}}\left(C^{*}(G, A, \alpha)\right) \cong K_{0}(B)$ as $\mathbb{Z}[G]$-modules. Applying this result to the suspension $S A$, we obtain the same isomorphism for $K_{1}$ as well. By Takai duality 27, there is an isomorphism $B \cong A \otimes K\left(l^{2}(G)\right)$ which intertwines the action $\beta$ with the tensor product of $\alpha$ and an inner action on $K\left(l^{2}(G)\right)$. It is therefore immediate that $K_{*}(B) \cong K_{*}(A)$ as $\mathbb{Z}[G]$-modules.

We immediately get a realization theorem using type $\mathrm{I} \mathrm{C}^{*}$-algebras.

Theorem 4.5. Let $G_{*}$ be a countable $\mathbb{Z}_{2}$-graded $R\left(\mathbb{Z}_{2}\right)$-module. Then there exists a separable type I $\mathrm{C}^{*}$-algebra $A$, and an action $\alpha$ of $\mathbb{Z}_{2}$ on $A$, such that $K_{*}^{\mathbb{Z}_{2}}(A) \cong G_{*}$ as $R\left(\mathbb{Z}_{2}\right)$-modules.

Proof. Identify $R\left(\mathbb{Z}_{2}\right)$ with $\mathbb{Z}\left[\widehat{\mathbb{Z}}_{2}\right]$ as discussed before Lemma 4.4 Since $\widehat{\mathbb{Z}}_{2} \cong \mathbb{Z}_{2}$, we can use Proposition 3.3 to find a separable type $\mathrm{I}^{*}$-algebra $B$ and an action $\beta: \widehat{\mathbb{Z}}_{2} \rightarrow \operatorname{Aut}(B)$ such that $K_{*}(B) \cong G_{*}$ as $R\left(\mathbb{Z}_{2}\right)$-modules. Let $A=C^{*}\left(\widehat{\mathbb{Z}}_{2}, B, \beta\right)$, equipped with the dual action $\alpha=\widehat{\beta}$ of $\mathbb{Z}_{2}$. Lemma 4.4 implies that $K_{*}^{\mathbb{Z}_{2}}(A) \cong G_{*}$ as $R\left(\mathbb{Z}_{2}\right)$-modules. Clearly $A$ is separable, and Theorem 4.1 of [21] implies that $A$ is type $\mathrm{I}$.

For the realization theorem using Kirchberg algebras, we need another lemma.

Lemma 4.6. Let

$$
A_{0} \stackrel{\rho_{1}}{\longrightarrow} A_{1} \stackrel{\rho_{2}}{\longrightarrow} A_{2} \stackrel{\rho_{3}}{\longrightarrow} \cdots
$$

be a direct system of $\mathrm{C}^{*}$-algebras in which each $A_{n}$ is a finite direct sum of Kirchberg algebras satisfying the Universal Coefficient Theorem. Suppose that for each $n$ the partial map determined by $\rho_{n}$ from any summand of $A_{n-1}$ to any other summand of $A_{n}$ is nonzero. Then the direct limit of this system is again a Kirchberg algebra satisfying the Universal Coefficient Theorem.

Proof. Let $A=\underline{\lim } A_{n}$. Clearly $A$ is separable and nuclear. The condition that all the partial maps be nonzero, together with the simplicity of all the summands, guarantees that the algebraic direct limit of the $A_{n}$ is simple. A standard argument now shows that $A$ is simple. It is easy to check, using standard direct limit methods, that $A$ has real rank zero and that every nonzero projection in $A$ is properly infinite. (For the second statement, use the fact that every projection in $A$ is equivalent to a projection in the algebraic direct limit.) Therefore $A$ is purely infinite simple. Finally, $A$ satisfies the Universal Coefficient Theorem because the class of such algebras is closed under direct limits. This is essentially Proposition 2.3(b) of [23], in view of Theorem 4.1 of [23] and its converse (the converse being trivial). 
Theorem 4.7. Let $G_{*}$ be a countable $\mathbb{Z}_{2}$-graded $R\left(\mathbb{Z}_{2}\right)$-module. Then there exists a unital Kirchberg algebra $A$, and an action $\alpha$ of $\mathbb{Z}_{2}$ on $A$, such that $K_{*}^{\mathbb{Z}_{2}}(A) \cong G_{*}$ as $R\left(\mathbb{Z}_{2}\right)$-modules, and such that $C^{*}\left(\mathbb{Z}_{2}, A, \alpha\right)$ is a Kirchberg algebra.

Proof. Identify $R\left(\mathbb{Z}_{2}\right)$ with $\mathbb{Z}\left[\widehat{\mathbb{Z}}_{2}\right]$ as discussed before Lemma 4.4] Let $\tau \in \widehat{\mathbb{Z}}_{2}$ be the nontrivial element.

First suppose that the action of $\tau$ on the $R\left(\mathbb{Z}_{2}\right)$-module $G_{*}$ is nontrivial. Since $\widehat{\mathbb{Z}}_{2} \cong \mathbb{Z}_{2}$, we can use Theorem 4.3 to find a unital Kirchberg algebra $B$ and an action $\beta: \widehat{\mathbb{Z}}_{2} \rightarrow \operatorname{Aut}(B)$ such that $K_{*}(B) \cong G_{*}$ as $R\left(\mathbb{Z}_{2}\right)$-modules. Let $A=C^{*}\left(\widehat{\mathbb{Z}}_{2}, B, \beta\right)$, equipped with the dual action $\alpha=\widehat{\beta}$ of $\mathbb{Z}_{2}$. Lemma 4.4 implies that $K_{*}^{\mathbb{Z}_{2}}(A) \cong G_{*}$ as $R\left(\mathbb{Z}_{2}\right)$-modules. Moreover, $\beta_{\tau}$ is outer (because it is nontrivial on $\mathrm{K}$-theory), so Corollary 4.4 of [12] implies that $A$ is a Kirchberg algebra. Moreover $C^{*}\left(\mathbb{Z}_{2}, A, \alpha\right) \cong$ $M_{2} \otimes B$ is also a Kirchberg algebra.

Now suppose that the action of $\tau$ on the $R\left(\mathbb{Z}_{2}\right)$-module $G_{*}$ is trivial. Choose a unital Kirchberg algebra $A_{0}$ satisfying the Universal Coefficient Theorem such that $K_{*}\left(A_{0}\right) \cong G_{*}$ as abelian groups. Let $\alpha^{(0)}: \mathbb{Z}_{2} \rightarrow \operatorname{Aut}\left(A_{0} \oplus A_{0}\right)$ be the action such that $\alpha_{\tau}^{(0)}(a, b)=(b, a)$. We identify the crossed product $C^{*}\left(\mathbb{Z}_{2}, A_{0} \oplus A_{0}, \alpha^{(0)}\right)$. Let $u \in C^{*}\left(\mathbb{Z}_{2}, A_{0} \oplus A_{0}, \alpha^{(0)}\right)$ be the canonical unitary of order two. Then there is an isomorphism $\sigma: C^{*}\left(\mathbb{Z}_{2}, A_{0} \oplus A_{0}, \alpha^{(0)}\right) \rightarrow M_{2}\left(A_{0}\right)$, determined by

$$
\sigma(a, b)=\left(\begin{array}{ll}
a & 0 \\
0 & b
\end{array}\right)
$$

for $a, b \in A_{0}$, and

$$
\sigma(u)=\left(\begin{array}{ll}
0 & 1 \\
1 & 0
\end{array}\right)
$$

The dual action is inner, so $K_{*}^{\mathbb{Z}_{2}}\left(A_{0} \oplus A_{0}\right) \cong G_{*}$ as $R\left(\mathbb{Z}_{2}\right)$-modules.

The algebra $A_{0} \oplus A_{0}$ is not a Kirchberg algebra. To remedy this, choose a nonzero projection $q \in A_{0}$ such that $[q]=0$ in $K_{0}\left(A_{0}\right)$. Set $p=1-q$, so that $[p]=\left[1_{A_{0}}\right]$ in $K_{0}\left(A_{0}\right)$. Choose (14]; or Theorem 4.1.3 and the proofs of Corollary 4.4.2 and Theorem 4.2.4 of [19]) unital homomorphisms $\varphi: A_{0} \rightarrow p A_{0} p$ and $\psi: A_{0} \rightarrow q A_{0} q$ such that

$$
A_{0} \stackrel{\varphi}{\longrightarrow} p A_{0} p \longrightarrow A_{0}
$$

is the identity on K-theory and $\psi_{*}=0$. Define a unital equivariant homomorphism $\rho: A_{0} \oplus A_{0} \rightarrow A_{0} \oplus A_{0}$ by $\varphi(a, b)=(\varphi(a)+\psi(b), \varphi(b)+\psi(a))$ for $a, b \in A_{0}$. Let $A$ be the direct limit of the system

$$
A_{0} \oplus A_{0} \stackrel{\rho}{\longrightarrow} A_{0} \oplus A_{0} \stackrel{\rho}{\longrightarrow} A_{0} \oplus A_{0} \stackrel{\rho}{\longrightarrow} \cdots .
$$

Then $A$ is a unital Kirchberg algebra by Lemma 4.6 Let $\alpha: \mathbb{Z}_{2} \rightarrow \operatorname{Aut}(A)$ be the direct limit action.

We next show that $K_{*}^{\mathbb{Z}_{2}}(A) \cong G_{*}$ as $R\left(\mathbb{Z}_{2}\right)$-modules. We do this by proving that $\rho_{*}: K_{*}^{\mathbb{Z}_{2}}\left(A_{0} \oplus A_{0}\right) \rightarrow K_{*}^{\mathbb{Z}_{2}}\left(A_{0} \oplus A_{0}\right)$ is an isomorphism and using Proposition 2.5.4 of [18] on the direct system and its suspension. We claim that the corresponding homomorphism

$$
\bar{\rho}: C^{*}\left(\mathbb{Z}_{2}, A_{0} \oplus A_{0}, \alpha^{(0)}\right) \rightarrow C^{*}\left(\mathbb{Z}_{2}, A_{0} \oplus A_{0}, \alpha^{(0)}\right)
$$

is, under the identification of the crossed product above, given by

$$
\bar{\rho}(x)=\left(\operatorname{id}_{M_{2}} \otimes \varphi\right)(x)+u\left(\operatorname{id}_{M_{2}} \otimes \psi\right)(x) u^{*} .
$$


It is enough to check this on the image of $A_{0} \oplus A_{0}$, namely the diagonal matrices, and on $u$; this is easy. It is immediate from the choice of $\varphi$ and $\psi$ that this map is the identity on K-theory.

It remains to show that $C^{*}\left(\mathbb{Z}_{2}, A, \alpha\right)$ is a Kirchberg algebra. This algebra is the direct limit of the system of crossed products

$C^{*}\left(\mathbb{Z}_{2}, A_{0} \oplus A_{0}, \alpha^{(0)}\right) \stackrel{\bar{\rho}}{\longrightarrow} C^{*}\left(\mathbb{Z}_{2}, A_{0} \oplus A_{0}, \alpha^{(0)}\right) \stackrel{\bar{\rho}}{\longrightarrow} C^{*}\left(\mathbb{Z}_{2}, A_{0} \oplus A_{0}, \alpha^{(0)}\right) \stackrel{\bar{\rho}}{\longrightarrow} \cdots$, which, by the computation at the beginning of this case, can be rewritten as

$$
M_{2}\left(A_{0}\right) \longrightarrow M_{2}\left(A_{0}\right) \longrightarrow M_{2}\left(A_{0}\right) \longrightarrow \cdots .
$$

The direct limit of this system is a Kirchberg algebra by Lemma 4.6

Remark 4.8. When the action of the nontrivial element of $\widehat{\mathbb{Z}}_{2}$ is nontrivial, we do not know whether the $\mathrm{C}^{*}$-algebra in Theorem 4.7 satisfies the Universal Coefficient Theorem.

\section{An explicit EXAMPle}

In this section, we write down a formula for an automorphism in the smallest nontrivial case of Theorem 4.3 This is the case $K_{0}(A) \cong \mathbb{Z}_{3}, K_{1}(A)=0$, and $\gamma_{0}$ is the unique nontrivial automorphism of $\mathbb{Z}_{3}$. Since $\gamma_{0}$ fixes only the identity element of $\mathbb{Z}_{3}$, the automorphism will exist only when $\left[1_{A}\right]=0$ in $K_{0}(A)$. Let $\mathcal{O}_{4}$ be the Cuntz algebra [6], with K-theory as computed in [7]. Then the unital Kirchberg algebra $A$ with this K-theory and satisfying the Universal Coefficient Theorem is $M_{3} \otimes \mathcal{O}_{4}$.

Example 5.1. We give an explicit formula, in terms of the standard generators, for an order two automorphism $\varphi$ of $A=M_{3} \otimes \mathcal{O}_{4}$ which is nontrivial on $K_{0}(A)$.

Let $s_{1}, s_{2}, s_{3}$, and $s_{4}$ be the standard generating isometries of $\mathcal{O}_{4}$, and define $p_{m}=s_{m} s_{m}^{*}$. Further let $e_{j, k}$, for $1 \leq j, k \leq 3$, be the standard matrix units of $M_{3}$, satisfying $e_{j, k} e_{k, l}=e_{j, l}$ etc. Then $\varphi$ is determined by the formulas:

$$
\begin{aligned}
& e_{1,1} \otimes 1 \mapsto f_{1,1}=\left(e_{2,2}+e_{3,3}\right) \otimes 1 \\
& e_{2,2} \otimes 1 \mapsto f_{2,2}=e_{1,1} \otimes\left(p_{1}+p_{2}\right) \\
& e_{3,3} \otimes 1 \mapsto f_{3,3}=e_{1,1} \otimes\left(p_{3}+p_{4}\right) \\
& e_{1,2} \otimes 1 \mapsto f_{1,2}=e_{2,1} \otimes s_{1}^{*}+e_{3,1} \otimes s_{2}^{*} \\
& e_{1,3} \otimes 1 \mapsto f_{1,3}=e_{2,1} \otimes s_{3}^{*}+e_{3,1} \otimes s_{4}^{*} \\
& e_{1,1} \otimes s_{1} \mapsto v_{1}=e_{2,2} \otimes s_{1}+e_{2,3} \otimes s_{2} \\
& e_{1,1} \otimes s_{2} \mapsto v_{2}=e_{2,2} \otimes s_{3}+e_{2,3} \otimes s_{4} \\
& e_{1,1} \otimes s_{3} \mapsto v_{3}=e_{3,2} \otimes s_{1}+e_{3,3} \otimes s_{2} \\
& e_{1,1} \otimes s_{4} \mapsto v_{4}=e_{3,2} \otimes s_{3}+e_{3,3} \otimes s_{4}
\end{aligned}
$$

One must check two things: that $\varphi$ is a homomorphism, and that $\varphi^{2}=\mathrm{id}_{A}$. For the first, one checks the following relations:

- $f_{1,1}, f_{2,2}$, and $f_{3,3}$ are orthogonal projections which sum to 1 .

- $f_{1, j} f_{1, j}^{*}=f_{1,1}$ and $f_{1, j}^{*} f_{1, j}=f_{j, j}$ for $j=2,3$.

- $v_{m}^{*} v_{m}=f_{1,1}$ for $1 \leq m \leq 4$ and $\sum_{m=1}^{4} v_{m} v_{m}^{*}=f_{1,1}$. 
The details of the computation are somewhat long, and are omitted.

To prove that $\varphi^{2}=\operatorname{id}_{A}$, one checks that $\varphi(\varphi(a))=a$ for each of the generators used above. Again, we omit the details.

\section{REFERENCES}

[1] H. Bass, Big projective modules are free, Illinois J. Math. 7(1963), 24-31.

[2] D. J. Benson and K. R. Goodearl, Periodic flat modules, and flat modules for finite groups, Pacific J. Math. 196(2000), 45-67.

[3] B. Blackadar, K-Theory for Operator Algebras, MSRI Publication Series 5, Springer-Verlag, New York, Heidelberg, Berlin, Tokyo, 1986.

[4] M. C. R. Butler, J. M. Campbell, and L. G. Kovács, Infinite rank integral representations of groups and orders of finite lattice type, in preparation.

[5] M. C. R. Butler and L. G. Kovács, Large lattices over classical orders of finite lattice type, draft preprint.

[6] J. Cuntz, Simple $C^{*}$-algebras generated by isometries, Commun. Math. Phys. 57(1977), 173185.

[7] J. Cuntz, K-theory for certain $C^{*}$-algebras, Ann. Math. 113(1981), 181-197.

[8] C. W. Curtis and I. Reiner, Representation Theory of Finite Groups and Associative Algebras, Interscience Publishers, New York, London, Sydney, 1962.

[9] G. A. Elliott and M. Rørdam, Classification of certain infinite simple $C^{*}$-algebras II, Comment. Math. Helv. 70(1995), 615-638.

[10] L. Fuchs, Infinite Abelian Groups. Vol. I, Academic Press, New York, London, 1970.

[11] L. K. Hua and I. Reiner, Automorphisms of the unimodular group, Trans. Amer. Math. Soc. 71(1951), 331-348.

[12] J. A. Jeong and H. Osaka, Extremally rich $C^{*}$-crossed products and the cancellation property, J. Austral. Math. Soc. (Series A) 64(1998), 285-301.

[13] E. Kirchberg, Commutants of unitaries in UHF algebras and functorial properties of exactness, J. reine angew. Math. 452(1994), 39-77.

[14] E. Kirchberg, The classification of purely infinite $C^{*}$-algebras using Kasparov's theory, preliminary preprint (3rd draft).

[15] A. Kumjian, On certain Cuntz-Pimsner algebras, preprint.

[16] S. Mac Lane, Categories for the Working Mathematician, Springer-Verlag Graduate Texts in Math. no. 5, Springer-Verlag, New York, Heidelberg, Berlin, 1971.

[17] G. K. Pedersen, C*-Algebras and their Automorphism Groups, Academic Press, London, New York, San Francisco, 1979.

[18] N. C. Phillips, Equivariant K-Theory and Freeness of Group Actions on $C^{*}$-Algebras, Springer-Verlag Lecture Notes in Math. no. 1274, Springer-Verlag, Berlin, Heidelberg, New York, London, Paris, Tokyo, 1987.

[19] N. C. Phillips, A classification theorem for nuclear purely infinite simple $C^{*}$-algebras, Doc. Math. 5(2000), 49-114 (electronic).

[20] M. Pimsner, A class of $C^{*}$-algebras generalizing both Cuntz-Krieger algebras and crossed products by $\mathbb{Z}$, pages 189-212 in: Free Probability Theory (Waterloo, ON, 1995), Fields Inst. Commun. vol. 12, Amer. Math. Soc., Providence, RI, 1997.

[21] M. A. Rieffel, Actions of finite groups on $C^{*}$-algebras, Math. Scand. 47(1980), 157-176.

[22] M. Rørdam and E. Størmer, Classification of nuclear $C^{*}$-algebras. Entropy in operator algebras, Springer-Verlag, Berlin, 2002.

[23] J. Rosenberg and C. Schochet, The Künneth theorem and the universal coefficient theorem for Kasparov's generalized K-functor, Duke Math. J. 55(1987), 431-474.

[24] C. Schochet, Topological methods for $C^{*}$-algebras II: geometric resolutions and the Künneth formula, Pacific J. Math. 98(1982), 443-458.

[25] C. Schochet, Topological methods for $C^{*}$-algebras III: axiomatic homology, Pacific J. Math. 114(1984), 399-445.

[26] R. G. Swan, Induced representations and projective modules, Ann. of Math. 71(1960), 552578.

[27] H. Takai, On a duality for crossed products of $C^{*}$-algebras, J. Funct. Anal. 19(1975), 25-39. 
Department of Mathematics, University of Georgia, Athens GA 30602-7403, USA.

E-mail address: djb@byrd.math.uga.edu

Department of Mathematics, University of Nevada, Reno NV 89557-0045, USA.

E-mail address: alex@unr.edu

Department of Mathematics, University of Oregon, Eugene OR 97403-1222, USA.

E-mail address: ncp@darkwing.uoregon.edu 